\title{
Over-the-scope clip vs epinephrine with clip for first-line hemostasis in non-variceal upper gastrointestinal bleeding: a propensity score match analysis
}

\section{다)(1) $\odot(9)$}

\author{
Authors \\ Santi Mangiafico ${ }^{1}$, Flavia Pigòn ${ }^{1}$, Helga Bertani ${ }^{1}$, Angelo Caruso ${ }^{1}$, Giuseppe Grande ${ }^{1}$, Costantino Sgamato ${ }^{2}$, Raffaele \\ Manta $^{3}$, Rita Conigliaro ${ }^{1}$
}

Institutions

1 Azienda Ospedaliero-Universitaria di Modena Ospedale Civile di Baggiovara, Gastroenterology and Digestive Endoscopy Unit, Modena, Italy

2 University of Naples Federico II, Department of Clinical Medicine and Surgery, Gastroenterology Unit, Naples, Italy

3 Azienda Ospedaliera di Perugia, Gastroenterology and Digestive Endoscopy Unit, Perugia, Italy

submitted 5.3.2019

accepted after revision 29.7.2019

\author{
Bibliography \\ DOI https://doi.org/10.1055/a-1005-6401 | \\ Endoscopy International Open 2020; 08: E50-E58 \\ (c) Georg Thieme Verlag KG Stuttgart · New York \\ eISSN 2196-9736
}

Corresponding author

Flavia Pigò, Azienda Ospedaliero-Universitaria di Modena Ospedale Civile di Baggiovara, Gastroenterology and

Digestive Endoscopy Unit, Viale giardini 1355, 41126

Modena, Italy

Fax: +39-059-3961216

flaviapigo@virgilio.it

\section{ABSTRACT}

Background and study aims The over-the-scope clip (OTSC) is a novel tool used to improve the maintenance of hemostasis for non-variceal upper gastrointestinal bleeding (NVUGIB); however, studies on the comparison with "conventional" techniques are lacking. In this study, we aimed to compare first-line endoscopic hemostasis achieved using conventional techniques with that achieved using OTSC placement for NVUGIB.

Patients and methods From January 2007 to March 2018, 793 consecutive patients underwent upper endoscopy with the hemostasis procedure. Among them, 327 patients were eligible for inclusion (112 patients had OTSC placement and 215 underwent conventional hemostasis). After propensity score matching and adjustment for confounding factors, 84 patients were stratified into the "conventional" group and 84 into the OTSC group. Patient characteristics and outcomes (rebleeding rate, mortality rate within 30 days, and adverse events) were compared between the two groups. Results In the unmatched cohort, hemostasis with OTSC was more frequent in cases of duodenal ulcers with Forrest la to Ila and in patients with a higher Rockall score compared with the "conventional group". In the matched cohort, $93 \%$ of the patients in the "conventional group" underwent hemostasis with epinephrine + through-the-scope clip. Rebleeding events were significantly less frequent in the OTSC group ( $8 \%$ vs $20 \%, 95 \% \mathrm{Cl} 3-16$ vs $12-30 ; P=0.02$ ); however, the mortality rate in the two groups was not significantly different ( $6 \%$ vs $2 \%, 95 \% \mathrm{Cl} 1-8$ vs $2-13 ; P=0.4$ ).

Conclusions OTSC is a safe and effective tool for achieving hemostasis, and we recommend its use as the first-line therapy for lesions with a high risk of rebleeding and in patients with a high risk Rockall score.

\section{Background}

During previous decades, the treatment and management of non-variceal upper gastrointestinal bleeding (NVUGIB) have substantially improved, with endoscopic treatment being the first-line modality. After the index endoscopy, rebleeding occurs in up to $20 \%$ of cases [1], with a mortality rate of $10 \%$ [2]. Recurrent bleeding after endoscopic therapy is associated with significant mortality, with a higher risk in older populations and those with multiple comorbidities. This trend may be attributable to the rising comorbidity in NVUGIB patients and the increasing use of antithrombotic drugs [3].

Therefore, there is a need to develop additional medical therapies that will improve the maintenance of hemostasis. The European Society of Gastrointestinal Endoscopy (ESGE) guidelines [4] recommend (strong recommendation, high- 
quality evidence) combining epinephrine injection with a second hemostasis modality (thermal contact, mechanical therapy, or injection of a sclerosing agent), especially for actively bleeding ulcers. The over-the-scope clip (OTSC ${ }^{\circledR}$, Ovesco Endoscopy $\mathrm{GmbH}$, Tübingen, Germany) is a novel tool that can securely hold a larger volume of tissue and to a greater depth with respect to the standard through-the-scope clip (TTS) [57]. To the best of our knowledge, there are no comparative studies on the efficacy of OTSC and other hemostatic methods for first-line hemostasis. Thus, we aimed to compare first-line endoscopic hemostasis achieved using conventional techniques versus that obtained using OTSC placement for NVUGIB.

\section{Materials and methods}

\section{Study population}

From January 2007 to March 2018, 793 consecutive patients underwent upper endoscopy with the hemostasis procedure for NVUGIB. The inclusion criteria were as follows: age $>18$ years, NVUGIB related to ulcers, Mallory Weiss lesion, Dieulafoy lesion, anastomotic bleeding, or angioectasia. The exclusion criteria were: incomplete clinical information, other causes of bleeding (post-sphincterotomy bleeding, post-polypectomy bleeding, malignancy, hemorrhagic gastritis, or watermelon stomach), or endoscopic hemostasis with only epinephrine injection because the ESGE recommends (strong recommendation and with high-quality evidence) that epinephrine injection therapy should not be used as endoscopic monotherapy. We collected data with regard to the following variables: age, sex, year of bleeding, number of major comorbidities (cardiac failure, ischemic heart disease, asthma, chronic obstructive pulmonary disease, diabetes mellitus, rheumatoid arthritis, liver failure, renal failure, disseminated malignancy, pneumonia, dementia, recent major operation, cerebrovascular disease, hematological malignancy, hypertension, trauma/burns, other cardiac disease, major sepsis, and/or other liver disease), anticoagulant/antithrombotic therapy, site of bleeding (esophagus, stomach, duodenum, and/or anastomosis), Forrest classification [8], hemostasis technique (epinephrine with/without TTS, OTSC, thermic device, or sclerosing agent) for the most severe lesion according to the Forrest classification, adverse events related to the hemostasis technique used, Rockall Score [9], Helicobacter pylori infection (assessed using biopsy or fecal antigen), rebleeding rate, rebleeding from a different site, rescue hemostasis technique (endoscopic, arterial embolization, or surgery), mortality rate within 30 days, and hospitalization (days). The study was approved by the Ethics Committee of the University of Modena on 10 May 2018 (Prot AOU 0011529/ 18).

\section{Description of the procedure}

All of the endoscopic procedures were performed in an inpatient setting, under anesthesia-assisted deep sedation by a single, skilled operator. Hemodynamically unstable patients were adequately resuscitated before they underwent upper endoscopy with crystalloid/colloid infusion and erythrocyte concentrate transfusion if needed. Patients with a non-cirrhosis related coagulopathy and with a prolonged prothrombin time with an international normalized ratio (INR) $>2.0$ were transfused with fresh frozen plasma. The use of prothrombin complex concentrate infusions was preferred for patients with serious/lifethreatening bleeding. We performed upper endoscopy once the INR was $<2.5$. Before endoscopy, the patients received an intravenous bolus of proton pump inhibitor (pantoprazole $80 \mathrm{mg}$ ), followed, if needed, by constant infusion ( $8 \mathrm{mg} /$ hour).

Early endoscopy (within 24 hours) was performed in all cases with either a diagnostic $(9.2-\mathrm{mm})$ or a therapeutic $(10-\mathrm{mm})$ endoscope (Pentax Medical, Tokyo, Japan). In order to achieve endoscopic hemostasis, in addition to epinephrine injection, we used thermal modalities (argon plasma coagulation, ERBE, $\mathrm{VIO}^{\circledR}$, Tuebingen, Germany), mechanical therapy with TTS clip (QuickClip2, Olympus ${ }^{\circledR}$, Tokyo, Japan; Resolution Clip, Boston Scientific ${ }^{\circledR}$, Natick MA, USA; DuraClip, ConMed ${ }^{\circledR}$, Greenwood, USA; SureClip, Micro-Tech ${ }^{\circledR}$, Anna Arbor, Mi, USA) and sclerosing agents, based on the choice of the endoscopist.

When we used the OTSCs, the endoscope was extracted and equipped with the OTSC system. The OTSC size (11 or $12 \mathrm{~mm}$ ) and type were chosen by the endoscopist. The 11-mm and 12mm OTSCs were used with both "diagnostic" ( $9.2 \mathrm{~mm}$, working channel $2.8 \mathrm{~mm}$ ) gastroscope and "therapeutic" (10 mm, working channel $3.7 \mathrm{~mm}$ ) gastroscope. The OTSC was deployed on the lesion either with suction or after tissue retraction into the cap with an anchor device. In addition, an injection of epinephrine solution was allowed (but not mandatory) before or after OTSC deployment.

\section{Outcomes and clinical data}

All of the data were retrospectively collected from medical records. The primary outcome was the rebleeding rate (defined as occurrence of hematemesis, aspiration of blood from the nasogastric tube, instability of arterial blood pressure of cardiac frequency, and a fall of $>2 \mathrm{~g} / \mathrm{dL}$ in the hemoglobin level) within 24 hours or within 30 days after hemostasis (immediate or late bleeding). Secondary outcomes were the mortality rate within 30 days and adverse events related to the hemostasis technique used.

\section{Statistical analyses}

A univariable analysis was conducted for all of the baseline characteristics presented in $>$ Table $\mathbf{1}$ and $>$ Table 2 . Variables that differed significantly between other hemostasis techniques and OTSC were used to create a propensity score so as to match the "conventional" group patients with the OTSC group (1:1). A propensity score is the probability that a unit with certain characteristics will be assigned to the treatment group (as opposed to the control group). The scores can be used to reduce or eliminate selection bias in observational studies by balancing the covariates between the treatment and control groups. Propensity score matching [10] creates sets of participants for the treatment and control groups. A matched set consists of at least one participant in the treatment group and one in the control group with similar propensity scores. The goal is to approximate a random experiment. Covariates to be included in the model were related to the out- 


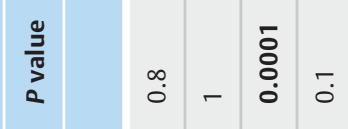

$\stackrel{\infty}{\circ}$

$\stackrel{\infty}{\circ}$

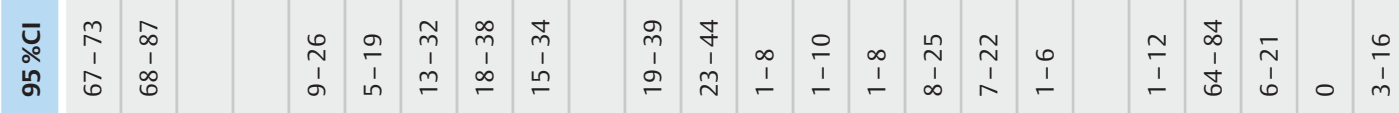

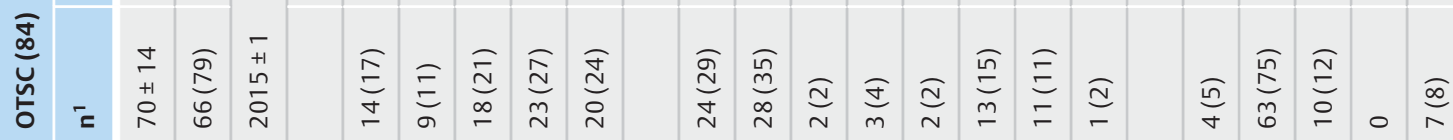

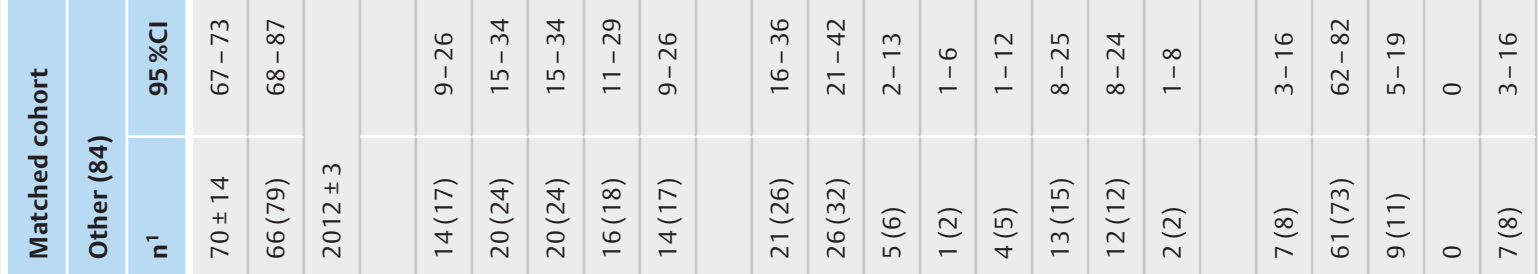

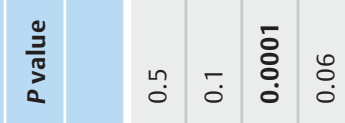

$\stackrel{m}{0}$

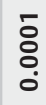

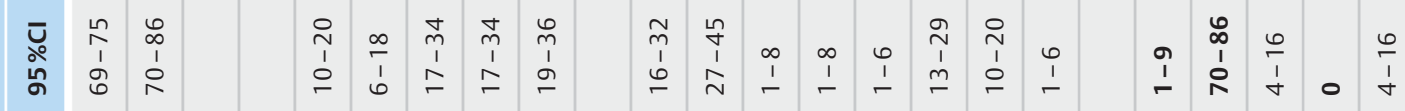

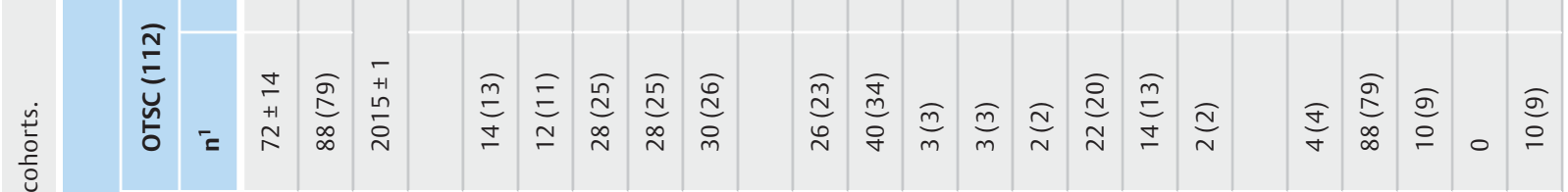

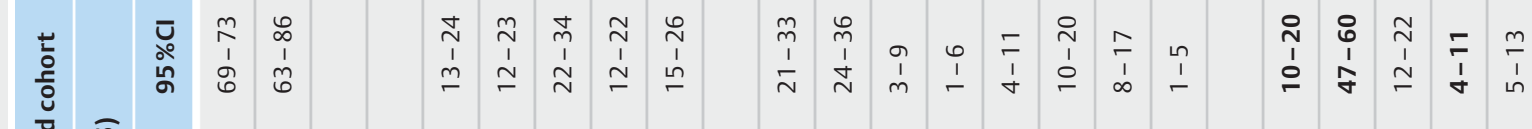

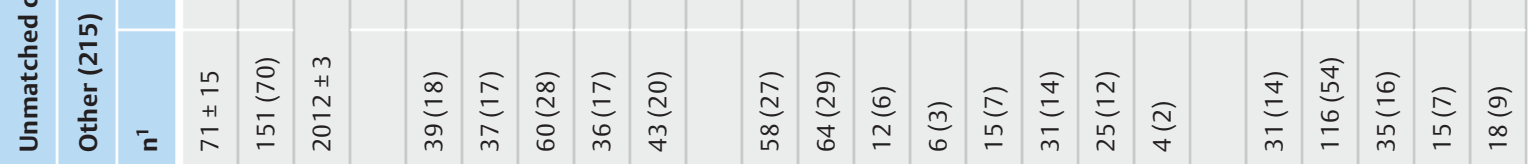

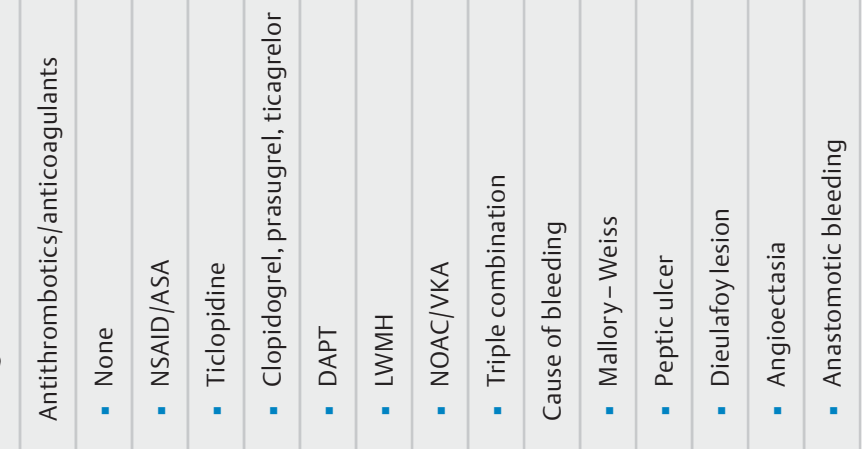




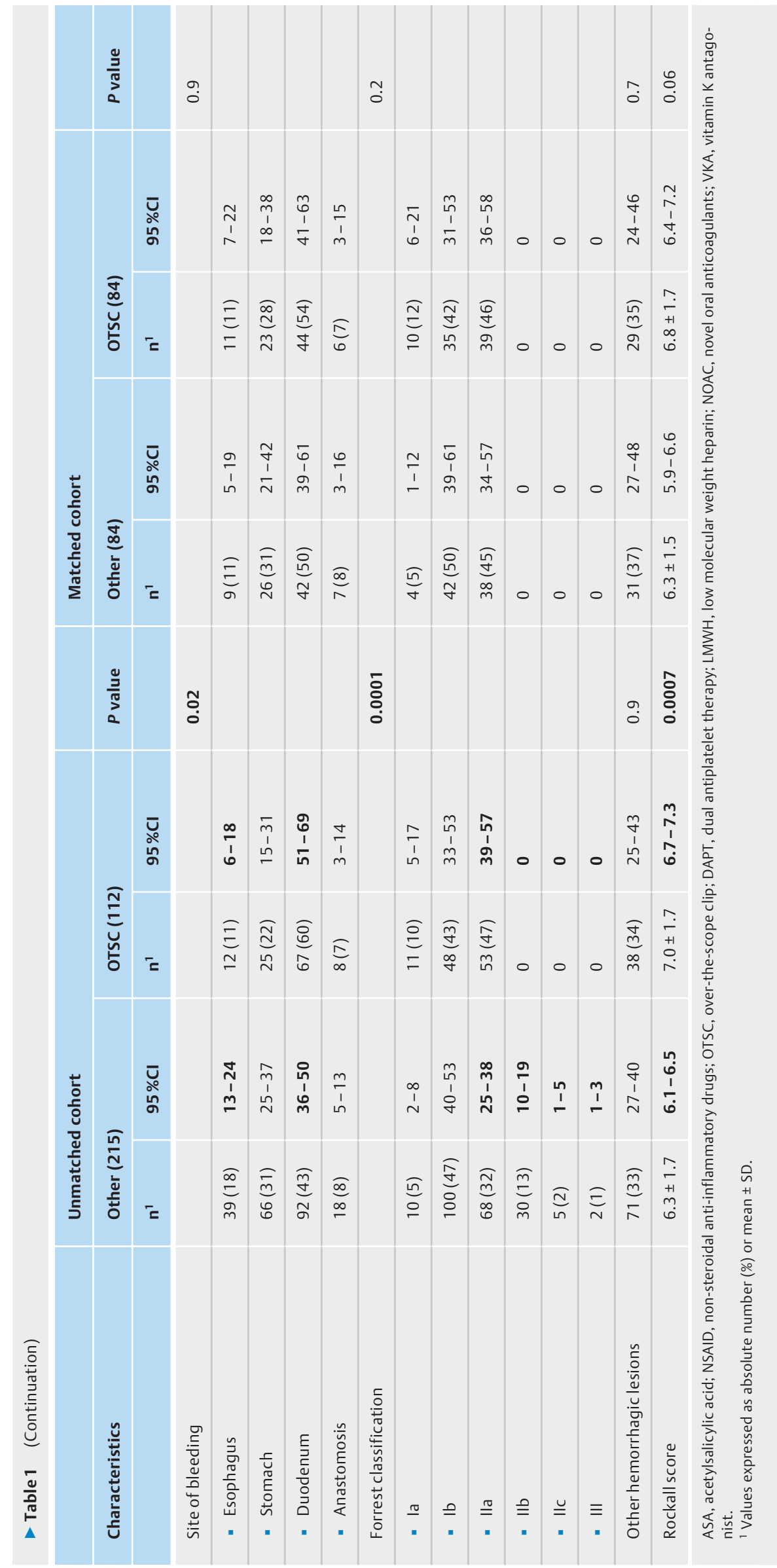


$\frac{\sqrt{2}}{\frac{3}{3}}$

$\stackrel{1}{0}$

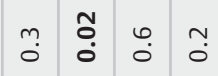

ナ.

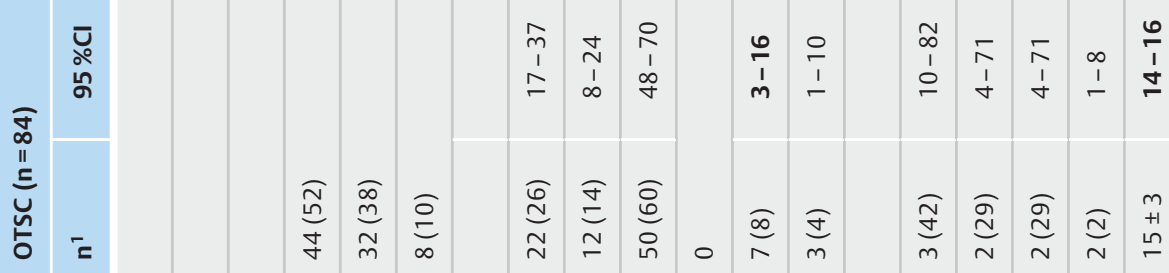

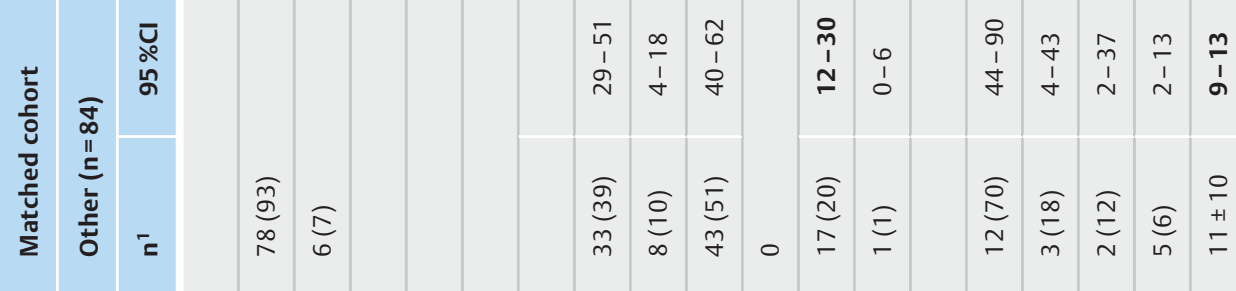
$\stackrel{\frac{0}{3}}{\frac{1}{2}}$
$\stackrel{0}{0}$

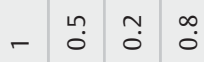
$\stackrel{\circ}{\circ}$

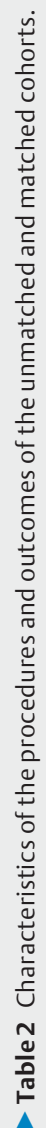

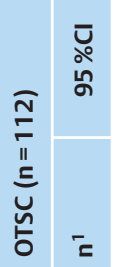

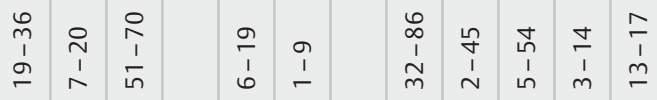

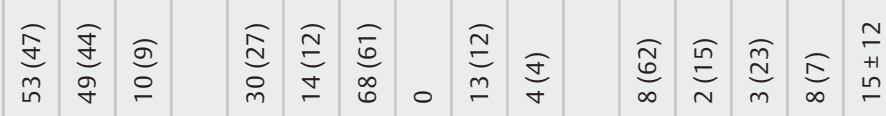

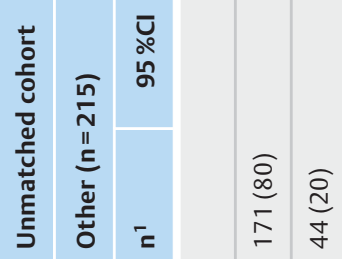

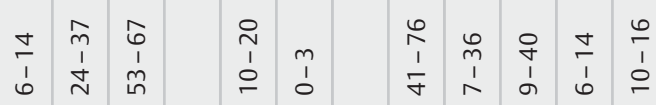

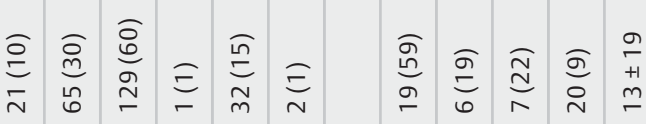

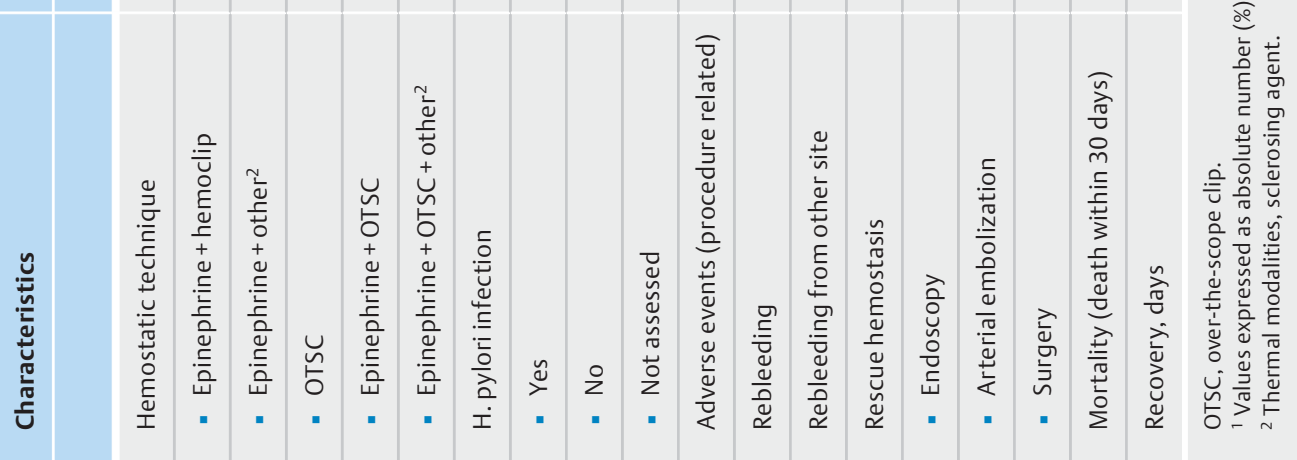




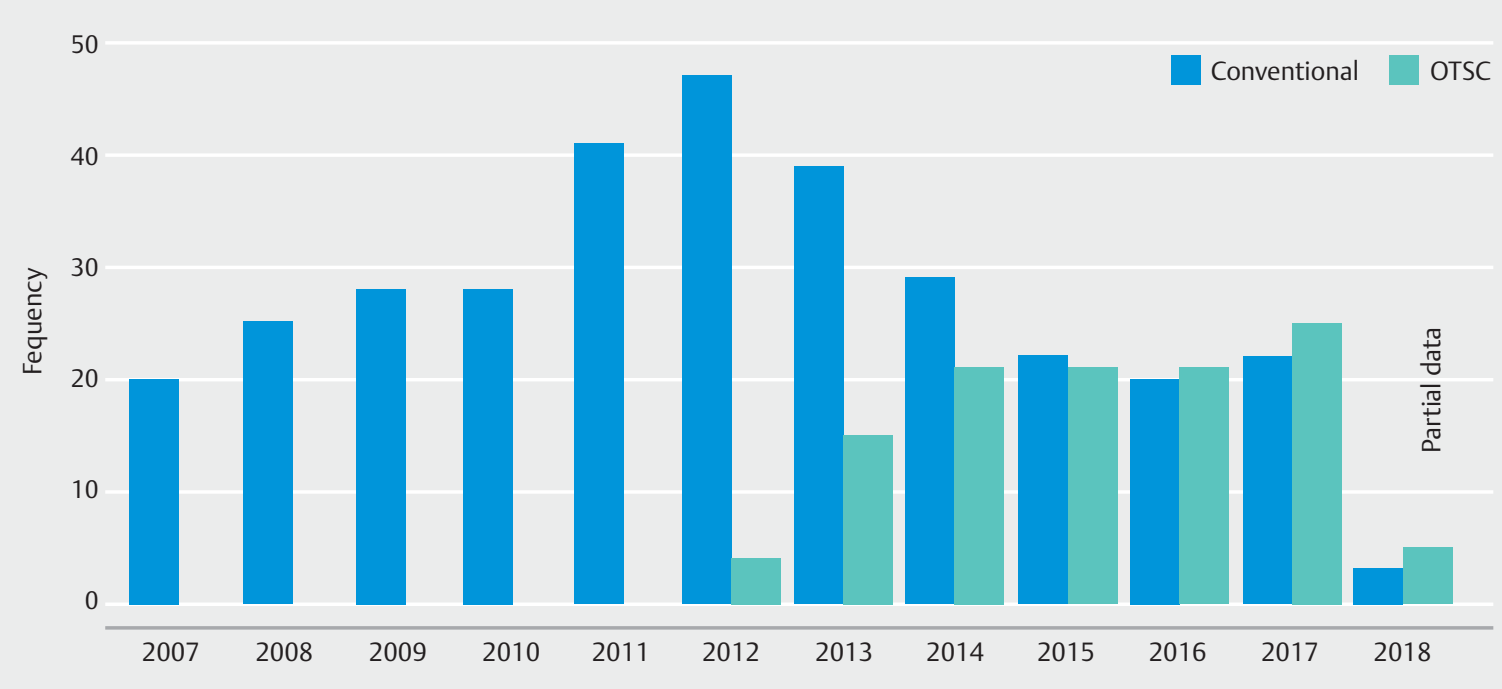

- Fig. 1 Number of procedures performed per study year. Only partial data available for 2018. OTSC, over-the-scope clip.

come but not to the exposure so as to increase the precision of the estimated exposure effect without increasing the bias [11]. Calculating a propensity score is an iterative process. The $t$ test (Stata statistical software, version 13, StataCorp, College Station, TX, United States) was used to determine whether each covariate was balanced within each block. Patients were matched using the nearest-neighbor method without replacement and with a caliper width equal to 0.1 .

Continuous data are expressed as mean \pm standard deviation values, while the categorical variables are presented as frequencies (\%). Continuous variables were compared using the Student's $t$ test, and categorical variables were compared with the $x^{2}$ or the Fisher's exact test, as appropriate. Stata version 13 was used for the statistical analyses.

\section{Results}

In total, 327 patients were eligible for inclusion. Of these, 112 patients had OTSC placement, and 215 underwent conventional hemostasis (epinephrine with/without TTS or thermic device or sclerosing agent). The OTSC group and the "conventional" group differed with respect to the year of bleeding, cause of bleeding, site of bleeding, Forrest classification, and Rockall score. The OTSC device was implemented in our endoscopic service ( $\mathbf{F i g}$. 1) from 2012 with an increase in the percentage until 2017 (for 2018, there is only partial data until March 2018). Utilization of hemostasis with OTSC was more frequent in duodenal ulcers with Forrest la to Ila and in patients in the "conventional" group with a higher Rockall score. Adverse events related to the procedure were only reported in a case with thermal (APC) hemostasis that worsened the bleeding. In order to mitigate the effects of measurable baseline confounders, patients were matched into 84 pairs using propensity score matching. Covariates included in the model were age, sex, number of comorbidities, cause of bleeding, site of bleeding, Forrest classification, and Rockall score. The population

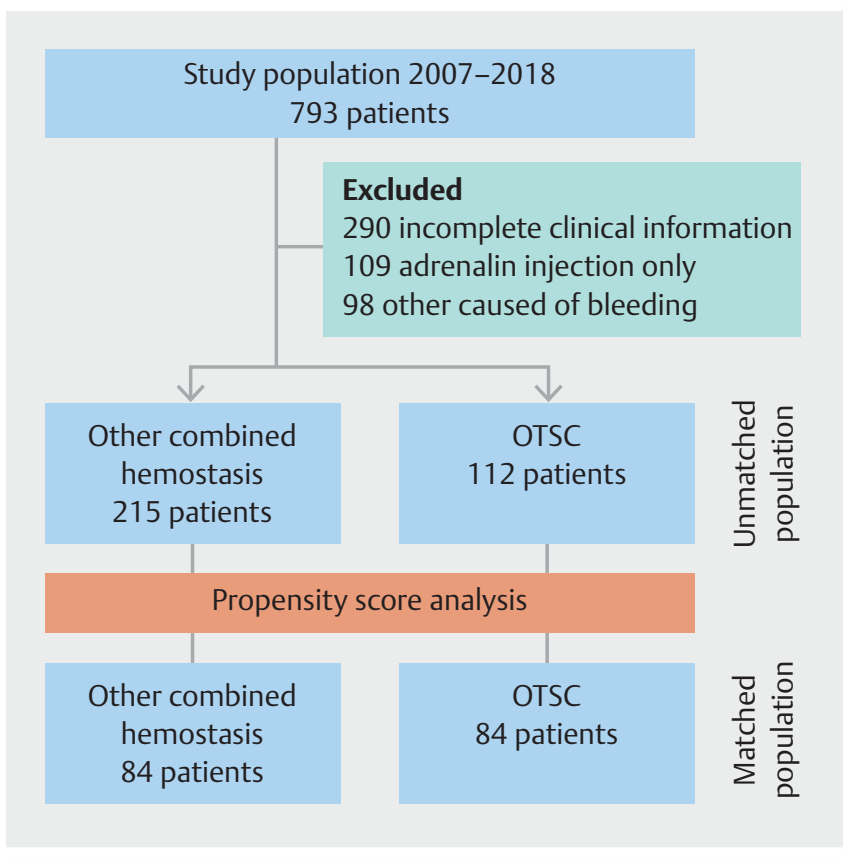

Fig. 2 Flow chart for the enrolled patients. OTSC, over-thescope clip.

flow chart is presented in $\mathbf{F i g . 2}$, and the baseline patient characteristics of the matched cohort are summarized in $>$ Table 1 and $>$ Table 2 . The kernel distribution of propensity scores before and after matching is shown in > Fig. 3a,b.

\section{Matched cohort}

The majority of the patients in both groups were men (79\%) aged $70 \pm 14$ years (mean $\pm S D$ ). At the time of bleeding, antithrombotics or anticoagulants were being used by most of the patients ( $74 \%$ of the conventional group and $71 \%$ of the OTSC 


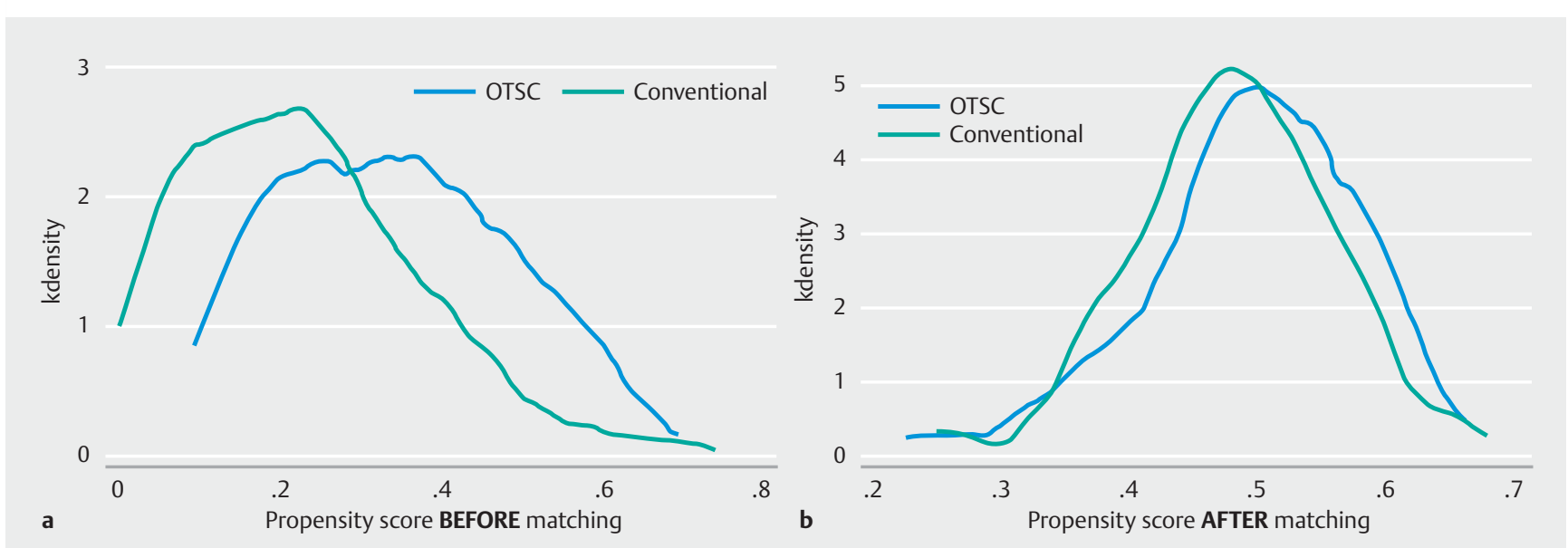

- Fig. 3 Kernel distribution of propensity scores: a before matching; b after matching.

group). The mean Rockall scores were $6.3 \pm 1.5$ and $6.8 \pm 1.7$ for the conventional and OTSC groups, respectively.

The most frequent site of bleeding was the duodenum, with the bleeding mainly being related to peptic ulcers with Forrest class la to Ila. Other hemorrhagic lesions with minor significance compared with the main bleeding source were diagnosed in $37 \%$ and $35 \%$ of cases in the "conventional" and OTSC groups, respectively. Among traditional hemostatic procedures, epinephrine + TTS was used in $93 \%$ of cases. On the other hand, OTSC was applied alone in $52 \%$ of all of the cases and in $38 \%$ of cases after the injection of epinephrine. No adverse events were reported. The H.pylori status was not assessed in $>50 \%$ of patients in both groups. Rebleeding events were less common in the OTSC group ( $20 \%$ vs $8 \%, 95 \%$ confidence interval (CI) $12-30$ vs $3-16$; $P=0.02)$; however, the mortality rate of the two groups was not significantly different (6\% vs $2 \%$; 95 $\% \mathrm{Cl} 2-13$ vs $1-8 ; P=0.4)$. Rescue hemostasis was mainly managed with another endoscopic procedure ( $42 \%$ vs $70 \%$ ) and less frequently with arterial embolization ( $29 \%$ vs $18 \%$ ) or surgery ( $29 \%$ vs $12 \%$ ) in the OTSC and "conventional" groups, respectively. Rescue hemostasis achieved with a second endoscopic procedure was managed with epinehprine + TTS, except for three patients, managed with other modalities (epinehprine + sclerosing agent). The length of hospital stay (days) was longer in the OTSC group than in the "conventional" group ( $15 \pm 3$ vs $11 \pm 10$ days, $95 \% \mathrm{Cl} 14-16$ vs $9-13$; $P$ value 0.03 )

\section{Discussion}

Despite the major advances in NVUGIB management over the past decade, including the prevention of peptic ulcer bleeding and high-dose proton pump inhibition, considerable morbidity, mortality, and health economic burdens persist. Of particular note are the rebleeding rates, one of the most crucial predictive factors of morbidity and mortality that has not significantly improved as evident from longitudinal data in the past 15 years [12-14]. Although several types of endoscopic treatment for NVUGIB have been described, including injection therapy, ther- mal coagulation, hemostatic clips, fibrin sealant (or glue), argon plasma coagulation, and combination therapy (typically injection of epinephrine combined with another treatment modality), relatively few comparative trials have been performed. Currently, most patients are being treated with either thermal coagulation therapy or hemostatic clips, with or without the addition of injection therapy.

In this study, we aimed to compare first-line endoscopic hemostasis, achieved using conventional techniques, with OTSC placement for NVUGIB in a matched cohort of patients. The OTSC system as a preliminary experience has been successfully used in patients with severe bleeding or deep wall lesions, or perforations of the gastrointestinal tract [14].

To date, clinical data on OTSC treatment for upper gastrointestinal bleeding is limited to case series and retrospective studies $[5,7,12-23]$. The technical success rate varies from $77.8 \%$ to $100 \%$ when OTSC is used as first-line therapy, with a rebleeding rate of $7.4-13.6 \%$. However, patient populations differ widely with respect to the bleeding source, bleeding severity, and previous therapy. Most studies include a limited sample size and lack a control group. In our study, we tried to overcome these limitations by using propensity score analysis to balance the confounding factors between the two groups. Our study showed the efficacy of OTSC as a first-line therapy in the management of NVUGIB. The rebleeding rate was acceptably low $(8 \%)$ in the OTSC group and was lower than that in the "conventional" group $(P=0.02)$. This finding is consistent with the previously reported rebleeding rates of $0-22 \%$ [24] and with the observation that rebleeding following OTSC placement occurs in up to $35 \%$ of patients receiving antithrombotic therapy [14]. In addition, data from the first randomized study found that OTSC application was more effective than standard hemostasis (TTS or thermal plus epinephrine) techniques as a rescue therapy in patients with recurrent bleeding peptic ulcer [6]. The bleeding-related mortality rate was $2 \%$ in the OTSC group, lower than that ( $8 \%$ ) in the control group and occurred in patients with important comorbidities. In our experience, death occurred in patients in whom the OTSC placement was 
impossible or who experienced rebleeding. Unfortunately, the fatal events occurred despite the interventional radiology or surgical approaches that were performed. This would appear consistent with mortality reported in other recent studies [7, 19, 23, 25].

In this study, we found and confirmed that, because of their lower rebleeding rate, OTSC devices are suitable for patients with duodenal ulcers with high Forrest classification status (Forrest la to Ila) and a high Rockall score.

The OTSC system, being a very contractile, super-elastic nickel titanium alloy, provides tissue apposition that is far superior to that of traditional clipping. Hemostasis is achieved by a combination of the following two mechanisms: (1) sealing the blood vessel; and (2) closing off an ulcer. However, the main mechanism appears to be that of "tissue compression" that occurs by compressing the surrounding tissue around the vessel. Although it is possible to close an ulcer by applying the OTSC directly on a bleeding vessel, it is believed that the above-mentioned "tissue compression" mechanism better explains the hemostatic mechanisms.

These characteristics enable us to overcome the limitations of TTS used in the compression of limited amounts of tissue, especially in the presence of scarred and hardened tissue or inflammatory mucosa with a hemostatic effect not sufficient for large-sized vessels. In addition, standard clips often detach from these lesions and induce more bleeding by lacerating the vessel. Nevertheless, we acknowledge the limitations of the OTSC system and agree with Asokkumar et al. [26] who have identified the following three common patterns that result in OTSC failure: (1) delayed closure of OTSC occurring in lesions with large caliber arteries and those with a deep fibrotic base; (2) shallow placement of OTSC resulting from inadequate suction or premature clip deployment; and (3) misplacement of OTSC because of poor visualization, difficult anatomy, and unstable endoscope position.

This study has certain limitations. First, because of the retrospective design, selection bias and confounding factors could affect the study validity. In order to limit the selection bias, all of the consecutive patients who underwent a hemostasis procedure for NVUGIB were considered for enrollment. However, owing to the retrospective nature of the study, complete information for all of the eligible patients was not available. Thus, 30 $\%$ of the eligible patients were excluded. In order to reduce the confounding factors in the two cohorts, propensity score analysis was applied to the study design. This strategy to reduce the confounding has an unavoidable limitation because it reduces the sample size. In our study, the post-hoc power (84 patients in each group, with a 0.05 alpha error and rebleeding rates of $20 \%$ in the conventional group and $8 \%$ in the OTSC group) is $61.2 \%$, which is slightly underpowered in comparison to the standard reference value $(80 \%)$. Second, propensity score matching may lead to increased covariate imbalance called the propensity score paradox [27]. Despite progressive pruning of the matched sets, the application of a caliper width of 0.1 should avoid pruning near the lowest region of the imbalance trend. However, it is possible that the nested variables influence the outcome. For example, it was not possible to assess the severity of the comorbidities, and this could affect the outcome. Moreover, the difference between the length of recovery in the two groups could be associated with the severity of comorbidities in the patients which was not scored in this study. Furthermore, the temporal relationship with the rebleeding rate was difficult to assess because, after the introduction of the OTSC device in our center in 2012, we preferred OTSC over TTS because of its observed, although empiric, major efficacy in hemostasis. Thus, most of the control patients were those treated before 2012, and during the study period, the skill level of the operators could have changed. Thus, we suggest using OTSC as the first-line treatment for lesions with a high risk of rebleeding in patients with a high risk Rockall score. Finally, randomized controlled trials and a formal cost-effectiveness analysis are needed to determine the impact of the first-line use of OTSC in patients with high risk NVUGIB.

\section{Competing interests}

\section{None}

\section{References}

[1] Sostres C, Lanas A. Epidemiology and demographics of upper gastrointestinal bleeding: prevalence, incidence and mortality. Gastrointest Endosc Clin N Am 2011; 21: 567 - 581

[2] Palmer K, Atkinson S, Donnelly M et al. Acute upper gastrointestinal bleeding: management. UK: National Institute for Health and Clinical Excellence; 2011: Available at https://www.nice.org.uk/guidance/ cg141/documents/acute-upper-gi-bleeding-full-guideline2

[3] Barada K, Abdul-Baki H, El Hajj II et al. Gastrointestinal bleeding in the setting of anticoagulation and antiplatelet therapy. J Clin Gastroenterol 2009; 43: 5-12

[4] Karstensen JG, Ebigbo A, Asbakken L et al. Nonvariceal upper gastrointestinal hemorrhage: European Society of Gastrointestinal Endoscopy (ESGE) Cascade Guideline. Endosc Int Open 2018; 06: E1256E1263

[5] Manno M, Mangiafico S, Caruso A et al. First-line endoscopic treatment with OTSC in patients with high-risk non-variceal upper gastrointestinal bleeding: preliminary experience in 40 cases. Surg Endosc 2016; 30: 2026 - 2029

[6] Schmidt A, Golder S, Goetz M et al. Over-the-scope clips are more effective than standard endoscopic therapy for patients with recurrent bleeding of peptic ulcers. Gastroenterology 2018; 155: 674-686

[7] Wedi E, Fischer A, Hochberger ] et al. Multicenter evaluation of firstline endoscopic treatment with the OTSC in acute non-variceal upper gastrointestinal bleeding and comparison with the Rockall cohort: the FLETRock study. Surg Endosc 2018; 32: 307 - 314

[8] Forrest JA, Finlayson ND, Shearmen DJ. Endoscopy in gastrointestinal bleeding. Lancet 1974; 17: $394-397$

[9] Rockall TA, Logan RF, Devlin HB et al. Risk assessment after acute upper gastrointestinal haemorrhage. Gut 1996; 38: 316-321

[10] Rosenbaum PR, Rubin DB. The central role of the propensity score in observational studies for causal effects. Biometrika 1983; 70: 41 - 55

[11] Brookhart MA, Patrick AR, Dormuth C et al. Adherence to lipid-lowering therapy and the use of preventive health services: an investigation of the healthy user effect. Am J Epidemiol 2007; 166: 348-354 
[12] Jairath V, Barkun AN. Improving outcomes from acute upper gastrointestinal bleeding. Gut 2012; 61: $1246-1249$

[13] Rockall TA, Logan RF, Devlin HB et al. Incidence of and mortality from acute upper gastrointestinal haemorrhage in the United Kingdom. Steering Committee and members of the National Audit of Acute Upper Gastrointestinal Haemorrhage. BMJ 1995; 311: 222-226

[14] Hearnshaw SA, Logan RF, Lowe D et al. Acute upper gastrointestinal bleeding in the UK: patient characteristics, diagnoses and outcomes in the 2007 UK audit. Gut 2011; 60: 1327-1335

[15] Kirschniak A, Subotova N, Zieker D et al. The Over-The-Scope Clip (OTSC) for the treatment of gastrointestinal bleeding, perforations, and fistulas. Surg Endosc 2011; 25: 2901 - 2905

[16] Wedi E, Gonzalez S, Menke D et al. One hundred and one over-thescope-clip applications for severe gastrointestinal bleeding, leaks and fistulas. World J Gastroenterol 2016; 22: $1844-1853$

[17] Mönkemüller K, Peter S, Toshniwal J et al. Multipurpose use of the "bear claw" (over-the-scope-clip system) to treat endoluminal gastrointestinal disorders. Dig Endosc 2014; 26: 350 - 357

[18] Skinner M, Gutierrez JP, Neumann $\mathrm{H}$ et al. Over-the-scope-clip is an effective rescue therapy for severe acute upper gastrointestinal bleeding. Gastrointest Endosc 2014; 79: AB143

[19] Richter-Schrag H-J, Glatz T, Walker $\mathrm{C}$ et al. First-line endoscopic treatment with over-the-scope clips significantly improves the primary failure and rebleeding rates in high-risk gastrointestinal bleeding: a single-center experience with 100 cases. World J Gastroenterol 2016; 22: $9162-9171$
[20] Manta R, Galloro G, Mangiavillano B et al. Over-the-scope clip (OTSC) represents an effective endoscopic treatment for acute $\mathrm{Gl}$ bleeding after failure of conventional techniques. Surg Endosc 2013; 27: $3162-3164$

[21] Baron TH, Song LM, Ross A et al. Use of an over-the-scope clipping device: multicenter retrospective results of the first U.S. experience (with videos). Gastrointest Endosc 2012; 76: 202 - 208

[22] Lamberts R, Koch A, Binner C et al. Use of over-the-scope clips (OTSC) for hemostasis in gastrointestinal bleeding in patients under antithrombotic therapy. Endosc Int Open 2017; 05: E324-E330

[23] Manta R, Mangiafico S, Zullo A et al. First-line endoscopic treatment with over-the-scope clips in patients with either upper or lower gastrointestinal bleeding: a multicenter study. Endosc Int Open 2018; 6: E1317-E1321

[24] Chan SM, Lau JYW. Can we now recommend OTSC as first-line therapy in case of non-variceal upper gastrointestinal bleeding? Endosc Int Open 2017; 5: E883-E885

[25] Brandler J, Buttar N, Baruah A et al. Efficacy of over-the-scope clips in management of high-risk gastrointestinal bleeding. Clin Gastroenterol Hepatol 2018; 16: 690-696

[26] Asokkumar R, Soetikno R, Sanchez-Yague A et al. Use of over-thescope-clip (OTSC) improves outcomes of high-risk adverse outcome (HR-AO) non-variceal upper gastrointestinal bleeding (NVUGIB). Endosc Int Open 2018; 6: E789-E796

[27] Ripollone JE, Huybrechts KF, Rothman KJ et al. Implications of the propensity score matching paradox in pharmacoepidemiology. Am J Epidemiol 2018; 187: 1951-1961 